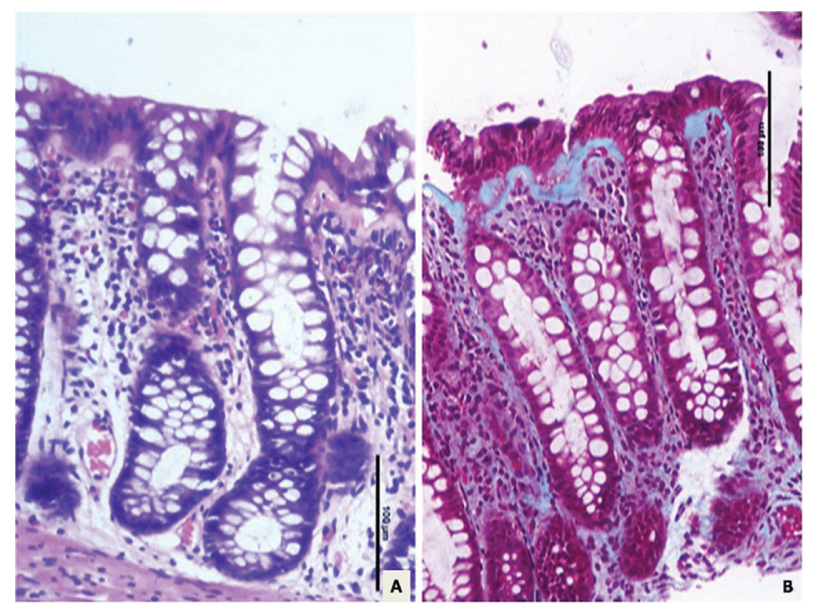

Figure 1. Photomicrograph of A showing mild to moderate increased intra-epithelial lymphocytes with thickened basement membrane B. shows the thickened basement membrane highlighted better in Masson's trichrome staining

Conclusions: Subclinical gut inflammation was significantly higher in PsA patients in comparison to $\mathrm{PsO}$ patients and is more prevalent among those with axial phenotype.

Acknowledgements: IRA

Disclosure of Interest: None declared

DOI: 10.1136/annrheumdis-2018-eular.3774

\section{SAT0303 PAIN MECHANISMS AND ULTRASONIC INFLAMMATORY ACTIVITY AS PROGNOSTIC FACTORS IN PATIENTS WITH PSORIATIC ARTHRITIS: RESULTS OF A DANISH PROSPECTIVE, EXPLORATORY COHORT STUDY}

P. Højgaard ${ }^{1,2}$, K. Ellegaard ${ }^{1}$, S.M. Nielsen ${ }^{1}$, R. Christensen ${ }^{1}$, J. Guldberg-Møller ${ }^{1}$, C. Ballegaard ${ }^{1,2}$, L. Dreyer ${ }^{1,2}$, P. Mease ${ }^{3}$, M. de Wit ${ }^{4}$, L. Skov ${ }^{5}$, B. Glintborg ${ }^{2,6}$, H. Bliddal ${ }^{1}$, E.M. Bartels ${ }^{1}$, K. Amris ${ }^{1}$, L.E. Kristensen ${ }^{1} .{ }^{1}$ The Parker Institute, Bispebjerg and Frederiksberg Hospital, Frederiksberg, Denmark; ${ }^{2}$ Center for Rheumatology and Spine diseases, Rigshospitalet Gentofte, Hellerup, Denmark; ${ }^{3}$ Swedish Medical Center, University of Washington, Seattle, USA; ${ }^{4}$ Department of Medical Humanities, Vrije University, Amsterdam, Netherlands; ${ }^{5}$ Department of Dermatology and Allergology, Herlev and Gentofte Hospital, Hellerup, Denmark; ${ }^{6}$ Center for Arthritis Research COPECARE, Rigshospitalet Glostrup, Glostrup, Denmark

Background: Pain is a major concern for patients with psoriatic arthritis (PsA) in spite of treatment with biologic/conventional disease modifying drugs (b/ csDMARDS). Studies on the prognostic role of pain mechanisms in PsA, are scarce.

Objectives: To investigate the presence of widespread non-arthritic pain (WP) in patients with PsA, and determine its relation to patient-reported, clinical- and ultrasound (US) disease measures, as well as to achievement of 4 months response to b/csDMARD therapy. Secondly, to study if US Colour Doppler activity (CD) at baseline is associated to treatment response.

Methods: A prospective cohort study was performed following a protocol. ${ }^{1} \mathrm{~Pa}$ tients initiating $\mathrm{b} / \mathrm{csDMARDs}$ for PsA were recruited from rheumatology clinics in Copenhagen. Clinical- and US examinations and patient-reported outcomes (PROs) were performed at baseline and after 4 months. WP was defined as a Widespread Pain Index $\geq 4$, and pain in $\geq 4 / 5$ regions. CD activity in selected joints, entheses and tendons (73 projections) was summed. Response was assessed by American College of Rheumatology 20\% (ACR20), Disease Activity in Psoriatic Arthritis 50\% (DAPSA 50) and Minimal Disease Activity (MDA). Main response analyses were based on intention to treat with non-responder imputation, and consisted of logistic regressions adjusting for gender and age.

Results: Of 123 screened patients, 69 were included. Of these, 24 (35\%) fulfilled the WP definition. At baseline (Table), WP was associated with worse PROs and composite scores, while CD activity and clinical measures were similar to those without WP. WP profile was significantly related to achievement of MDA at followup but not to other response criteria (table 1). Presence of CD activity at baseline was not significantly associated to 4 months response by any criteria, e.g. 19\%/ $31 \%$ with $C D=0 / C D>0$, reached ACR $20,(p=0.262)$.
Abstract SAT0303 - Table 1. Baseline characteristics

\begin{tabular}{lccc}
\hline Females, $\mathrm{n}(\%)$ & $15(63)$ & $24(53)$ & 0.634 \\
\hline PsA impact of Disease & $6.2(1.7)$ & $4.2(2.2)$ & $<0.001$ \\
(PsAID) & & & \\
HAQ-DI (0-3) & $1.2[0.9$ to 1.5$]$ & $0.6[0.3$ to 1.0$]$ & $<0.001$ \\
VAS Pain (0-100) & $68.8(19.2)$ & $44.5(27.6)$ & $<0.001$ \\
DAPSA (0-164) & $49.3(18.1)$ & $29.8(18.5)$ & $<0.001$ \\
SPARCC enthesitis (0-16) & $7.5(3.4)$ & $4.2(3.0)$ & $<0.001$ \\
CD score (0-200) & $1.0[0.0$ to 4.3$]$ & $1.0[0.0$ to 5.0$]$ & 0.686 \\
Swollen Joints (0-66) & $3.0[1.0$ to 6.3$]$ & $4.0[2.0$ to 8.0$]$ & 0.352 \\
Tender Joints (0-68) & $26.5[24.5$ to & $11.0[6.0$ to & $<0.001$ \\
& $37.0]$ & $20.0]$ & \\
4 months responses & & & \\
ACR20, $\mathrm{n}(\%)$ & $6(25)$ & $12(28)$ & 0.779 \\
DAPSA 50, n (\%) & $7(29)$ & $15(33)$ & 0.724 \\
MDA, n (\%) & $0(0)$ & $9(20)$ & 0.022 \\
\hline
\end{tabular}

Data as mean(SD) or median[IQR] P-value from T test/Mann Whitney U-test $/ \chi^{2}$ test,

Conclusions: WP was present in $1 / 3$ of patients, and associated with worse PROs, composite measures, and failure to achieve MDA at follow-up. Neither WP nor $C D$ at baseline was related to other response measures.

\section{REFERENCE}

Højgaard et al. BMJ Open 2006;6. doi:10.1136/bmjopen-2015-010650

Acknowledgements: Thanks to: The Oak foundation, Danish Rheumatism Association, Centre for Rheumatology Gentofte Hospital.

Disclosure of Interest: None declared

DOI: 10.1136/annrheumdis-2018-eular.1829

\section{SAT0304 PRECLINICAL PHASES OF PSORIATIC ARTHRITIS: A CROSS-SECTIONAL ULTRASONOGRAPHIC STUDY ON PSORIASIS AND PSORIATIC ARTHRALGIA PATIENTS}

A. Zabotti ${ }^{1}$, F. Zuliani ${ }^{1}$, E. Errichetti ${ }^{1}$, I. Tinazzi ${ }^{2}$, S. Sacco ${ }^{1}$, L. Quartuccio ${ }^{1}$, A. Zanetti ${ }^{3}$, A. Batticciotto ${ }^{4}$, O. De Lucia $^{5}$, L. Idolazzi ${ }^{6}$, G. Stinco ${ }^{1}$, A. Iagnocco ${ }^{7}$ S. De Vita ${ }^{1}{ }^{1}$ Rheumatology Clinic, "Santa Maria della Misericordia" University Hospital, Udine, Italy, Udine; ${ }^{2}$ Rheumatology Clinic, Ospedale Sacro Cuore Don Calabria, Verona; ${ }^{3}$ Epidemiology Unit, Italian Society of Rheumatology,

${ }^{4}$ Rheumatology Unit, Sacco University Hospital; ${ }^{5}$ Department of Rheumatology, ASST Centro traumatologico ortopedico G. Pini - CTO, Milan; ${ }^{6}$ Rheumatology Unit, University of Verona, Ospedale Civile Maggiore, Verona; ${ }^{7}$ Dipartimento di Scienze Cliniche e Biologiche, University of Turin, Turin, Italy

Background: Identify the preclinical phase of arthritis could be clinically relevant In this scenario, musculoskeletal ultrasonography (US) may play an important role, since it may detect subclinical disease. Psoriatic Arthritis (PsA) is a perfect disease to identify risk factors, since the risk pool group [i.e. patients with only cutaneous Psoriasis (Pso)] is known.

Objectives: To evaluate in Pso patients, with and without clinical arthralgia (CA) defined by the presence of joint pain without other clinical evidence of musculoskeletal inflammation: I) the prevalence of subclinical US inflammation in joints, enthesis, tendons and bursae; II) the prevalence of US structural damage; III) the relationship between US lesions and clinical data.

Methods: Cross-sectional prevalence study of US abnormalities in Pso patients with or without CA and healthy controls (HCs). Inclusion and exclusion criteria (e. g. osteoarthritis and fibromyalgia) are pre-defined. Forty-four joints (MCP, PIP and DIP joints, wrists, knees, MTP joints) and 12 enthesis (achilles, quadriceps, proximal and distal patellar, plantar aponeurosis and common extensor tendon enthesis) were scanned in each patient. US scans were performed using a ESAOTE MyLabClassC equipped with a high frequency linear probe. Active synovitis was defined by the presence of a grade $\geq 2$ for grey scale (GS) and $\geq 1$ for $P D$, while active enthesitis if there was hypoecogenity in GS and an entheseal PD signal ( $\leq 2 \mathrm{~mm}$ from bone insertion).

Results: Sixty-four Pso patients and $21 \mathrm{HCs}$ were included; globally 2816 joints and 768 entheses were scanned. Twenty-three out of 64 (35.9\%) Pso patients displayed CA. Baseline characteristics are reported in table 1. Active synovitis was found, in at least one joint, in 20/64 (31.3\%) Pso patients and 0/21 HCs $(p=0.002)$, while active enthesitis in $14 / 64(21.9 \%)$ Pso patients and $0 / 21 \mathrm{HCs}$ $(p=0.017)$. No significant differences were found for active synovitis or enthesitis between the two subgroups of Pso, with or without CA. In the Pso cohort, 5/23 (23.8\%) patients with CA and 2/41 (5\%) without CA displayed tenosynovitis or paratenonitis $(p=0.042)$. Furthermore, active synovitis, as well as GS-synovitis $\geq 2$, was associated with higher NAPSI $(9.7 \pm 8.0$ vs $4.6 \pm 7.1, \mathrm{p}=0.04$ for $\mathrm{GS} \geq 2$ ), 